TRANSACTIONS OF THE

AMERICAN MATHEMATICAL SOCIETY

Volume 350, Number 5, May 1998, Pages 2129-2140

S 0002-9947(98)02014-5

\title{
THE AVERAGE EDGE ORDER OF TRIANGULATIONS OF 3-MANIFOLDS WITH BOUNDARY
}

\author{
MAKOTO TAMURA
}

\begin{abstract}
Feng Luo and Richard Stong introduced the average edge order $\mu_{0}(K)$ of a triangulation $K$ and showed in particular that for closed 3-manifolds $\mu_{0}(K)$ being less than 4.5 implies that $K$ is on $S^{3}$. In this paper, we establish similar results for 3-manifolds with non-empty boundary; in particular it is shown that $\mu_{0}(K)$ being less than 4 implies that $K$ is on the 3-ball.
\end{abstract}

\section{INTRODUCTION}

Let $M$ be a compact, connected 3-manifold and $K$ a triangulation of $M$ (for the definition, see [2]). Note that we distinguish a triangulation from a cell complex consisting of 3 -simplices, that is, such a cell complex is a triangulation when the intersection of any two simplices is actually a face of each of them (Lemma 4.1). Suppose $M$ is closed. Then the average edge order $\mu_{0}(K)$ of $K$ is defined to be $3 F_{0}(K) / E_{0}(K)$, where $E_{0}(K)$ and $F_{0}(K)$ are the numbers of edges and faces in $K$, respectively. This is equal to the average of the orders of edges of $K$, where the order of an edge is the number of faces incident to that edge. Feng Luo and Richard Stong showed in [1] that for a closed 3-manifold $M$, the average edge order being small implies that the topology of $M$ is fairly simple and restricts the triangulation $K$ of $M$. In fact, they proved the following theorem.

Theorem $1.1([1])$. Let $K$ be any triangulation of a closed connected 3-manifold M. Then

(a) $3 \leq \mu_{0}(K)<6$, and equality holds if and only if $K$ is the triangulation of the boundary of a 4-simplex.

(b) For any $\varepsilon>0$, there are triangulations $K_{1}$ and $K_{2}$ of $M$ such that $\mu_{0}\left(K_{1}\right)<$ $4.5+\varepsilon$ and $\mu_{0}\left(K_{2}\right)>6-\varepsilon$.

(c) If $\mu_{0}(K)<4.5$, then $K$ is a triangulation of $S^{3}$. There are an infinite number of distinct such triangulations, but for any constant $c<4.5$ there are only finitely many triangulations $K$ with $\mu_{0}(K) \leq c$.

(d) If $\mu_{0}(K)=4.5$, then $K$ is a triangulation of $S^{3}, S^{2} \times S^{1}$, or $S^{2} \tilde{\times} S^{1}$. Furthermore, in the last two cases, the triangulations can be described.

For a related study, see [4]. The author showed similar results for compact 3manifolds with non-empty boundary in [3], by employing $\mu(K)=3 F(K) / E(K)$, where $E(K)$ (resp. $F(K)$ ) is the number of edges (resp. faces) in $K$. In particular, $B^{3}, D^{2} \times S^{1}$, and $D^{2} \tilde{\times} S^{1}$ are characterized. And then, the referee and Professor S. Kojima kindly suggested to the author that the more natural generalization of

Received by the editors November 10, 1994 and, in revised form, September 26, 1996.

1991 Mathematics Subject Classification. Primary 57Q15; Secondary 57M15.

(c)1998 American Mathematical Society 
the average edge order of closed manifolds to that of manifolds with boundary is to count simplices on the boundary with weight $1 / 2$. Their suggestions are based upon the fact that the average edge order is a geometric interpretation of the curvature in terms of a global average.

According to them, we modify the definition of the average edge order as follows. Put $E_{0}(K)=E_{i}(K)+E_{\partial}(K) / 2$ and $F_{0}(K)=F_{i}(K)+F_{\partial}(K) / 2$, where $E_{i}(K)$ (resp. $\left.F_{i}(K)\right)$ is the number of edges (resp. faces) in int $K=K \backslash \partial K$, and $E_{\partial}(K)$ (resp. $\left.F_{\partial}(K)\right)$ is the number of edges (resp. faces) on $\partial K$. Then we define the average edge order $\mu_{0}(K)$ of a triangulation $K$ of a 3-manifold with boundary to be $3 F_{0}(K) / E_{0}(K)$. This is the average of the "orders" of edges of $K$, where we modify the definition of the order of an edge $e$ on $\partial K$ as follows: The order of $e$ is twice the number of triangles incident to that edge, where we count triangles on the boundary with weight $1 / 2$. Note that this is equal to the order of the copy of $e$ in the canonical double of $K$. Explicitly, let $D(K)$ be the canonical double of $K$. Then $D(K)$ is a cell decomposition of a closed manifold into a union of 3-simplices. Though it may not be a triangulation, we define the order of an edge of $D(K)$ and the average edge order $\mu_{0}(K)$ as in the beginning of this introduction. Then the order of an edge $e$ of $\partial K$ in $K$ is equal to that of the copy of $e$ in $D(K)$, and we have $\mu_{0}(K)=\mu_{0}(D(K))$.

By using this average edge order, we have the following theorem.

Theorem 1.2. Let $K$ be any triangulation of a compact connected 3-manifold $M$ with non-empty boundary. Then

(a) $2 \leq \mu_{0}(K)<6$, and equality holds if and only if $K$ is the triangulation of one 3-simplex.

(b) For any rational number $r$ with $4<r<6$, there is a triangulation $K^{\prime}$ of $M$ such that $\mu_{0}\left(K^{\prime}\right)=r$.

(c) If $\mu_{0}(K)<4$, then $K$ is a triangulation of $B^{3}$. There are an infinite number of distinct such triangulations, but for any constant $c<4$ there are only finitely many triangulations $K$ with $\mu_{0}(K) \leq c$.

(d) If $\mu_{0}(K)=4$, then $K$ is a triangulation of $B^{3}, D^{2} \times S^{1}$, or $D^{2} \tilde{\times} S^{1}$. Furthermore, in the last two cases, the triangulations can be described.

\section{Proof of Theorem $1.2(a)$ And $(b)$}

The proof of Theorem $1.2(a)$ is easy. Since each edge has order at least 2 , we have $\mu_{0}(K) \geq 2$. Since the upper bound in Theorem $1.1(a)$ is valid for any cell complex of a closed 3-manifold consisting of 3-simplices, we have $\mu_{0}(K)=\mu_{0}(D(K))<6$. Finally, suppose $\mu_{0}(K)=2$. If an edge $e$ is in int $K$, then the order of $e$ is at least 3 because $K$ is a triangulation. Thus each edge of $K$ is on $\partial K$ and its order is 2. Hence we see $K$ consists of only one 3 -simplex. This completes the proof of Theorem $1.2(a)$.

The proof of $(b)$ is similar to that of Theorem $2(b)$ in [3], so we give only its outline. Let $r=q / p$ be a rational number with $4<r<6$, where $p$ and $q$ are relatively prime integers. We can see that there is a triangulation $K$ of $M$ such that $K$ contains edges $e_{1}, e_{2}$, and $e_{3}$ which satisfy the following conditions:

(1) $e_{1}$ lies in $\partial K$ and the order of $e_{1}$ is 2 ,

(2) $e_{2}$ and $e_{3}$ lie in $i n t K$ and the orders of them are $\alpha$ and $\alpha+1$, respectively, for some integer $\alpha$ satisfying $q / p<6 \alpha /(\alpha+1)$, and 
(3) $s t\left(e_{i}\right)(1 \leq i \leq 3)$ have mutually disjoint interiors, where $s t(e)$ is the star neighborhood of $e$ in $K$.

Let $K^{\prime}$ be the stellar subdivision of $K$ obtained by adding $2 l, m$, and $n$ vertices in the interior of $e_{1}, e_{2}$, and $e_{3}$, respectively. Then

$\mu_{0}\left(K^{\prime}\right)=3\left\{F_{0}(K)+4 l+2 \alpha m+2(\alpha+1) n\right\} /\left\{E_{0}(K)+3 l+(\alpha+1) m+(\alpha+2) n\right\}$.

All that we still have to do is to check the following, which is left to the reader: there exist non-negative integers $l, m$, and $n$ satisfying $\mu\left(K^{\prime}\right)=q / p$, that is,

$3(4 p-q) l+\{6 \alpha p-(\alpha+1) q\} m+\{6(\alpha+1) p-(\alpha+2) q\} n=q E_{0}(K)-3 p F_{0}(K)$.

To check this, note that $F_{0}(K)=2 T_{0}(K)$ is even, where $T_{0}(K)$ is the number of tetrahedra of $K$.

\section{The first SteP of the Proof of Theorem $1.2(c)$ And $(d)$}

To prove $(c)$ and $(d)$ of Theorem 1.2, it is convenient to define

$$
\xi(K)=3 F_{0}(K)-4 E_{0}(K)
$$

for a triangulation $K$ of a compact manifold. As $\mu_{0}(K)=4\left(3 F_{0}(K) / 4 E_{0}(K)\right)$, $\mu_{0}(K)<4$ (resp. $\left.\mu_{0}(K)=4\right)$ is equivalent to $\xi(K)<0(\operatorname{resp} . \xi(K)=0)$. We prove the following theorem.

Theorem 3.1. Let $K$ be a triangulation of a compact, connected 3-manifold $M$ with non-empty boundary. Then

(a) $\xi(K) \geq-6$, and equality holds if and only if $K=\natural^{k} \Delta^{3}$ for some positive integer $k$.

(b) If $M$ is not homeomorphic to $B^{3}$, then $\xi(K) \geq 0$, equality holds if and only if $K=\natural^{k} \Delta^{3} / \sim$, where $\sim$ denotes the identification of two faces on the boundary whose star neighborhoods have no intersection.

Here $\bigsqcup^{k} \Delta^{3}$ denotes the boundary connected sum of tetrahedra along their faces.

By this theorem, we have the first half of (c) and (d) of Theorem 1.2. By assuming it, we prove the latter half of Theorem 1.2 (c). Since we count simplices on the boundary with weight $1 / 2$, we see that $F_{0}\left(K \downarrow \Delta^{3}\right)=F_{0}(K)+F_{0}\left(\Delta^{3}\right)=F_{0}(K)+2$ and $E_{0}\left(K \natural \Delta^{3}\right)=E_{0}(K)+E_{0}\left(\Delta^{3}\right)-3 / 2=E_{0}(K)+3 / 2$, and hence $\xi\left(K \bigsqcup \Delta^{3}\right)=\xi(K)$ for any triangulation $K$. Therefore we have $\xi\left(\natural^{k} \Delta^{3}\right)=\xi\left(\Delta^{3}\right)=-6<0$ for any positive integer $k$, and hence there are infinitely many triangulations $K$ of $B^{3}$ with $\mu_{0}(K)<4$. For any constant $c<4$, suppose $\mu_{0}(K)=3 F_{0}(K) / E_{0}(K)<c$. Then $\xi(K)=3 F_{0}(K)-4 E_{0}(K)<(c-4) E_{0}(K)$. Since $\xi(K) \geq-6$ by Theorem 3.1 (a), we have $-6<(c-4) E_{0}(K)$. This gives the bound of the number of edges in any

triangulation $K$ with $\mu_{0}(K)<c$, that is, $E_{0}(K)<6 /(4-c)$. Therefore there are only finitely many such triangulations.

\section{Preliminaries for the Proof of Theorem 3.1}

In this section, we prepare several lemmas. The following two lemmas were proved in [1].

Lemma 4.1 (Lemma 1 in [1]). Let $D$ be a cell decomposition of an n-manifold $M$ into a union of simplices. Then $D$ fails to be a triangulation if and only if there are two simplices of $D$ with the same boundary. 
We will perform an operation on a triangulation which will be called contraction of an edge of the triangulation. Explicitly, given an edge $[x y]$ in the triangulation $K$, we let $K^{\prime}$ be the cell decomposition obtained from $K$ by removing all simplices containing $[x y]$ and identifying two $r$-simplices $\left[x v_{1} \ldots v_{r}\right]$ and $\left[y v_{1} \ldots v_{r}\right]$ if and only if $\left[x y v_{1} \ldots v_{r}\right]$ is an $(r+1)$-simplex of $K$. The following lemma tells us when this procedure produces a triangulation. A subcomplex $\Sigma$ of $K$ is called an essential $\partial \Delta^{k}$ if $\Sigma$ is isomorphic to the simplicial complex $\partial \Delta^{k}$ and does not bound a $k$ simplex in $K$.

Lemma 4.2 (Lemma 2 in [1]). Contraction of the edge [xy] in the triangulation $K$ produces a triangulation $K^{\prime}$ if and only if $[x y]$ lies in no essential $\partial \Delta^{k}$.

For the set of triangulations of compact 3-manifolds, we introduce the lexicographical order with respect to $\left(b_{1}(K),|\partial K|, T_{0}(K)\right)$, where $b_{1}(K)$ is the first Betti number of the polyhedron of $K,|\partial K|$ is the number of connected components of the polyhedron of $\partial K$, and $T_{0}(K)$ is the number of tetrahedra of $K$; that is, $K^{\prime}<K$ if and only if one of the following holds;

(1) $b_{1}\left(K^{\prime}\right)<b_{1}(K)$,

(2) $b_{1}\left(K^{\prime}\right)=b_{1}(K)$ and $\left|\partial K^{\prime}\right|<|\partial K|$,

(3) $b_{1}\left(K^{\prime}\right)=b_{1}(K),\left|\partial K^{\prime}\right|=|\partial K|$, and $T_{0}\left(K^{\prime}\right)<T_{0}(K)$.

In the proof of the theorem, we need to study $\Sigma$, an essential $\partial \Delta^{3}$ in $K$. To remove it, we will produce two kinds of operations. The following lemma describes the effects of these operations on $\xi(K)$.

Lemma 4.3. Let $\Sigma$ be an essential $\partial \Delta^{3}$ in $K$.

(1) Suppose $\Sigma$ is contained in $\partial K$. Let $K^{\prime}$ be the cell complex obtained by gluing a tetrahedron to $K$ along $\Sigma$. Then $K^{\prime}$ is a triangulation satisfying $\xi\left(K^{\prime}\right)=\xi(K)-6$ and $K^{\prime}<K$.

(2) Suppose $\Sigma$ is not contained in $\partial K$. Let $K^{\prime}$ be the cell complex obtained from $K$ by cutting along $\Sigma$ and gluing two tetrahedra along the images of $\Sigma$ (see Figure 1 ). Then $K^{\prime}$ is a triangulation satisfying $\xi\left(K^{\prime}\right)=\xi(K)-12$. Moreover, any connected component, say $K_{0}$, of $K^{\prime}$ satisfies $K_{0}<K$.

Proof. Lemma 4.1 shows that $K^{\prime}$ is a triangulation in both cases. The equality in (1) follows from the equalities $F_{0}\left(K^{\prime}\right)=F_{0}(K)+2$ and $E_{0}\left(K^{\prime}\right)=E_{0}(K)+3$. (Recall that we count cells on the boundary with weight $1 / 2$.) Since $b_{1}\left(K^{\prime}\right)=b_{1}(K)$ and $\left|\partial K^{\prime}\right|=|\partial K|-1$, we have $K^{\prime}<K$. This completes the proof of (1).

To prove the equality in (2), we use the following notation. For a subspace $X$ of $K$ consisting of open simplices of $K$, put $E_{0}(X, K)=E_{i}(X, K)+(1 / 2) E_{\partial}(X, K)$ and $F_{0}(X, K)=F_{i}(X, K)+(1 / 2) F_{\partial}(X, K)$, where $E_{i}(X, K)\left(\right.$ resp. $\left.F_{i}(X, K)\right)$ is the number of edges (resp. faces) of $X$ in int $K$ and $E_{\partial}(X, K)$ (resp. $F_{\partial}(X, K)$ ) is the number of edges (resp. faces) of $X$ on $\partial K$. Let $\xi(X, K)=3 F_{0}(X, K)-4 E_{0}(X, K)$. Then $\xi(K)=\xi\left(K^{(2)}, K\right)$, where $K^{(2)}$ is the 2-skeleton of $K$.

Since we count simplices on the boundary with weight $1 / 2$, we have

$$
F_{0}(\Sigma, K)+F_{0}(\Sigma \cap \partial K, K)=4
$$

and

Thus we have

$$
E_{0}(\Sigma, K)+E_{0}(\Sigma \cap \partial K, K)=6 .
$$

$$
\xi(\Sigma, K)+\xi(\Sigma \cap \partial K, K)=-12 .
$$



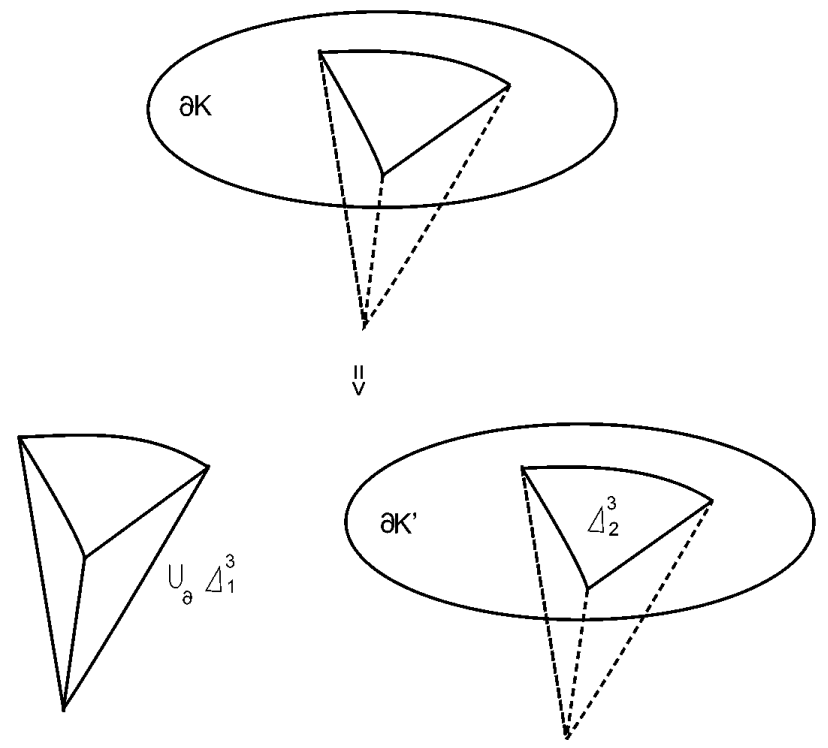

FiguRE 1

Note that the "image" of $\Sigma$ in $K^{\prime}$ consists of two components, say $\Sigma_{1}$ and $\Sigma_{2}$, where $\Sigma_{1} \cong \Sigma$ and $\Sigma_{2} \cong \operatorname{cl}(\Sigma \backslash \partial K)$. Let $\tilde{\Sigma}_{i} \subset K^{\prime}$ be the boundary of the 3 -simplex glued to $\Sigma_{i}$ in the construction of $K^{\prime}(i=1,2)$. Then we have

$$
\xi\left(\tilde{\Sigma}_{i}, K^{\prime}\right)+\xi\left(\tilde{\Sigma}_{i} \cap \partial K^{\prime}, K^{\prime}\right)=-12 \quad(i=1,2) .
$$

For each simplex $\sigma$ in $\Sigma \cap \partial K$, there is a unique image $\tilde{\sigma}$ of $\sigma$ in $K^{\prime}$ such that $\tilde{\sigma} \in \partial K^{\prime}$. Conversely, each simplex $\tilde{\sigma}$ in $\left(\tilde{\Sigma_{1}} \cup \tilde{\Sigma_{2}}\right) \cap \partial K^{\prime}$ is the image of some simplex $\sigma$ in $\partial K$. Hence we have

$$
\xi(\Sigma \cap \partial K, K)=\xi\left(\tilde{\Sigma_{1}} \cap \partial K^{\prime}, K^{\prime}\right)+\xi\left(\tilde{\Sigma_{2}} \cap \partial K^{\prime}, K^{\prime}\right) .
$$

Since there is a 1 to 1 correspondence between the simplices of $K^{(2)} \backslash \Sigma$ and those of $K^{\prime(2)} \backslash\left(\tilde{\Sigma_{1}} \cup \tilde{\Sigma_{2}}\right)$, we have

$$
\xi\left(K^{(2)} \backslash \Sigma, K\right)=\xi\left(K^{\prime(2)} \backslash\left(\tilde{\Sigma_{1}} \cup \tilde{\Sigma_{2}}\right), K^{\prime}\right) .
$$

Hence

$$
\begin{aligned}
\xi & \left(K^{\prime}\right) \\
& =\xi\left(K^{\prime(2)}, K^{\prime}\right) \\
& =\xi\left(K^{\prime(2)} \backslash\left(\tilde{\Sigma_{1}} \cup \tilde{\Sigma_{2}}\right), K^{\prime}\right)+\xi\left(\tilde{\Sigma_{1}}, K^{\prime}\right)+\xi\left(\tilde{\Sigma_{2}}, K^{\prime}\right) \\
& =\xi\left(K^{\prime(2)} \backslash\left(\tilde{\Sigma_{1}} \cup \tilde{\Sigma_{2}}\right), K^{\prime}\right)-\xi\left(\tilde{\Sigma_{1}} \cap \partial K^{\prime}, K^{\prime}\right)-\xi\left(\tilde{\Sigma_{2}} \cap \partial K^{\prime}, K^{\prime}\right)-24 \quad \text { by }(4.2) \\
& =\xi\left(K^{(2)} \backslash \Sigma, K\right)-\xi(\Sigma \cap \partial K, K)-24 \quad \text { by }(4.3) \text { and }(4.4) \\
& =\xi\left(K^{(2)} \backslash \Sigma, K\right)+\xi(\Sigma, K)-12 \quad \text { by }(4.1) \\
& =\xi\left(K^{(2)}, K\right)-12 \\
& =\xi(K)-12 .
\end{aligned}
$$


If $K^{\prime}$ is connected, then $b_{1}\left(K^{\prime}\right)=b_{1}(K)-1$ and hence $K^{\prime}<K$. Suppose $K^{\prime}$ is disconnected and let $K_{0}$ be a component of $K^{\prime}$. Then $b_{1}\left(K_{0}\right) \leq b_{1}(K),\left|\partial K_{0}\right| \leq|\partial K|$, and $T_{0}\left(K_{0}\right)<T_{0}(K)$, and hence $K^{\prime}<K$. This completes the proof of (2).

The next lemma is obtained as a corollary of Theorem 3 in [1].

Lemma 4.4 (Theorem 3 in [1]). Let $K$ be any triangulation of a closed connected 3-manifold $M$. Then

(1) $3\left(F_{0}(K)-4\right) /\left(E_{0}(K)-6\right) \geq 4.5$, and equality holds if and only if $K$ is a triangulation of $S^{3}$ as $\sharp^{k} \partial \Delta^{4}$ for some $k \geq 1$.

(2) If $M \neq S^{3}$, then $\mu_{0}(K)=3 F_{0}(K) / E_{0}(K) \geq 4.5$, equality holds if and only if $K$ is a triangulation of $S^{2} \times S^{1}$ or $S^{2} \tilde{\times} S^{1}$ obtained from $\sharp^{k} \partial \Delta^{4}$ by removing two open 3-simplices and gluing along their boundaries.

Lemma 4.5. Let $K$ be any triangulation of a closed connected 3-manifold $M$. Then

(1) $\xi(K)>-12$.

(2) If $M \neq S^{3}$, then $\xi(K)>0$.

Proof. (1) We see from Lemma 4.4 (1) that

$$
\xi(K)=3 F_{0}(K)-4 E_{0}(K) \geq E_{0}(K) / 2-15 .
$$

Since $K$ has more than one tetrahedron and $K$ is closed, $E_{0}(K)>6$. Thus $\xi(K) \geq$ $E_{0}(K) / 2-15>-12$. 0 .

(2) If $M \neq S^{3}$, then by Lemma $4.4(2), \xi(K)=3 F_{0}(K)-4 E_{0}(K) \geq E_{0}(K) / 2>$

\section{Proof of the inequality part of Theorem 3.1}

In this section, we prove the inequality part of Theorem 3.1, and we postpone the proof of the equality part until the next section. Suppose the inequality part of Theorem 3.1 does not hold, that is, there is a triangulation $K$ of a compact connected 3-manifold $M$ with non-empty boundary which satisfies one of the following conditions.

(1) $\xi(K)<-6$,

(2) $\xi(K)<0$ and $M \neq B^{3}$.

Let $\Gamma$ be the set of counterexamples of the inequalities in the theorem and $K$ a minimal element of $\Gamma$ in the lexicographical order with respect to the collection $\left(b_{1}(K),|\partial K|, T_{0}(K)\right)$. We shall prove the following claims.

Claim 5.1. $K$ has no essential $\partial \Delta^{3}$.

Claim 5.2. There are no edges of order 3 of $K$.

Claim 5.3. Any tetrahedron of $K$ has at most one face on $\partial K$.

Claim 5.4. Suppose $K$ satisfies the conclusions of Claims 5.2 and 5.3. Then $\xi(K) \geq 0$.

By Claim 5.4, $K$ is not an element of $\Gamma$, a contradiction. Therefore, by assuming the above claims, we have the inequality part of Theorem 3.1.

5.1. Proof of Claim 5.1. Suppose $K$ contains $\Sigma$, an essential $\partial \Delta^{3}$. Suppose $\Sigma \subset \partial K$. Put $K^{\prime}=K \cup_{\Sigma} \Delta^{3}$ as in Lemma 4.3 (1). Then $\xi\left(K^{\prime}\right)=\xi(K)-6$ and $K^{\prime}<K$. If $\partial K^{\prime} \neq \emptyset$, then $K^{\prime} \in \Gamma$. This contradicts the minimality of $K$. So $K^{\prime}$ is a triangulation of a closed 3-manifold. If $K$ is a triangulation of $B^{3}$, then $K^{\prime}$ is a triangulation of $S^{3}$ and we have $\xi(K)=\xi\left(K^{\prime}\right)+6>-6$ by Lemma 4.5 (1). 
This contradicts the assumption $K \in \Gamma$. If $K$ is not a triangulation of $B^{3}, K^{\prime}$ is not a triangulation of $S^{3}$ and we have $\xi(K)=\xi\left(K^{\prime}\right)+6>6$ by Lemma 4.5 (2), a contradiction.

Suppose $\Sigma$ is not contained in $\partial K$. Let $K^{\prime}$ be the cell complex obtained from $(K, \Sigma)$ as in Lemma $4.3(2)$, and let $\Sigma_{1}$ and $\Sigma_{2}$ be the subcomplexes of $K^{\prime}$ as in the proof of Lemma 4.3. By Lemma $4.3(2), \xi\left(K^{\prime}\right)=\xi(K)-12<-12$ and $K^{\prime}<K$. If $K^{\prime}$ is connected, then $\partial K^{\prime} \neq \emptyset$ and hence $K^{\prime}$ is an element of $\Gamma$. This contradicts the minimality of $K$. So $K^{\prime}$ is disconnected. Let $K_{i}$ be the component of $K^{\prime}$ containing $\Sigma_{i}$ for $i=1,2$. Then $K_{i}<K(i=1,2), \partial K_{2} \neq \emptyset$, and we have the following equality by Lemma $4.3(2)$ :

$$
\xi(K)=\xi\left(K_{1}\right)+\xi\left(K_{2}\right)+12 .
$$

Suppose $\partial K_{1} \neq \emptyset$. Since $K \in \Gamma$, we have $\xi(K)<0$ and hence some $K_{i}$ satisfies $\xi\left(K_{i}\right)<-6$. Since $\partial K_{i} \neq \emptyset, K_{i}$ is an element of $\Gamma$, a contradiction.

Suppose $\partial K_{1}=\emptyset$. If $K_{1}$ is not a triangulation of $S^{3}, \xi\left(K_{1}\right)>0$ by Lemma 4.5 (2). Then by (5.1), $\xi\left(K_{2}\right)<\xi\left(K_{1}\right)+\xi\left(K_{2}\right)=\xi(K)-12<-12$, and hence $K_{2}$ is an element of $\Gamma$, a contradiction. If $K_{1}$ is a triangulation of $S^{3}$, then $K_{2}$ is a triangulation of the given manifold $M$. Since $\xi\left(K_{1}\right)>-12$ by Lemma 4.5 , we get $\xi(K)>\xi\left(K_{2}\right)$ by (5.1). So $K_{2}$ is an element of $\Gamma$, a contradiction.

5.2. Proof of Claim 5.2. This follows from Claim 5.1 and the following lemma.

Lemma 5.5. Let $K$ be a triangulation of a compact connected 3-manifold $M$ with non-empty boundary which does not contain an essential $\partial \Delta^{3}$. If $K$ contains an edge of order 3 , then there is another triangulation $K^{\prime}$ of the given manifold $M$ such that $K^{\prime}<K$ and $\xi\left(K^{\prime}\right)=\xi(K)-2$.

Proof of Lemma 5.5. Let [xy] be an edge of order 3 in $K$. Then $K$ has the subcomplex $C$ as illustrated in Figure 2 (a). We can obtain another cell complex $K^{\prime}$ of $M$ by replacing $C$ with $C^{\prime}$ as illustrated in Figure $2(\mathrm{~b})$. We have $\xi\left(K^{\prime}\right)=\xi(K)-2$. Let $\{a, b, c\}$ be the vertices of the link of $[x y]$ in $K$. Suppose there is a face $[a b c]$ in $K$. Since $K$ does not contain an essential $\partial \Delta^{3}, K$ contains the tetrahedra [xabc] and $[y a b c]$. Hence $K$ is a triangulation of $S^{3}$. This contradicts $\partial K \neq \emptyset$. Thus $K$ does not have the face $[a b c]$ and hence $K^{\prime}$ is a triangulation by Lemma 4.1. Since $b_{1}\left(K^{\prime}\right)=b_{1}(K),\left|\partial K^{\prime}\right|=|\partial K|$, and $T_{0}\left(K^{\prime}\right)=T_{0}(K)-1$, we have $K^{\prime}<K$.

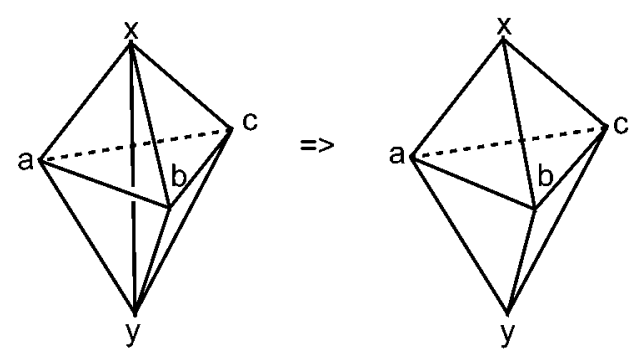

FIGURE 2 


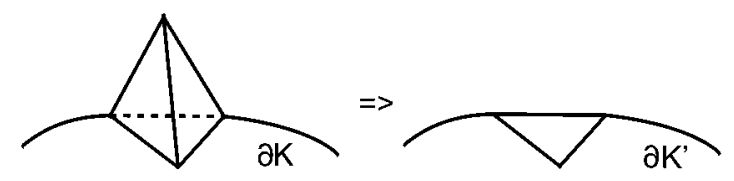

FiguRE 3

5.3. Proof of Claim 5.3. If there is a tetrahedron of $K$ which has all its faces on $\partial K$, then $K=\Delta^{3}$ and hence $\xi(K)=-6$. This contradicts the assumption $K \in \Gamma$.

For the case when $K$ has a tetrahedron $\Delta_{0}^{3}$ which contains two or three faces on $\partial K$, we have the following lemma.

Lemma 5.6. Let $K$ be a triangulation of a compact connected 3-manifold $M$ with non-empty boundary which does not contain an essential $\partial \Delta^{3}$. Suppose $K$ is not $\Delta^{3}$. If $K$ has a tetrahedron $\Delta_{0}^{3}$ which contains just two or three faces on $\partial K$, then there is a triangulation $K^{\prime}$ with $K^{\prime}<K$ which satisfies the following:

(1) If $\Delta_{0}^{3} \cap \partial K$ contains just three faces, then $K^{\prime}$ is a triangulation of $M$ and $\xi\left(K^{\prime}\right)=\xi(K)$.

(2) Suppose $\Delta_{0}^{3} \cap \partial K$ contains just two faces.

(2.1) Suppose further that $\Delta_{0}^{3}$ has an edge in int $K$. Then $K^{\prime}$ is a triangulation of $M$ and $\xi\left(K^{\prime}\right)=\xi(K)-2$.

(2.2) Suppose that $\Delta_{0}^{3}=[x y z w]$ does not have an edge in intK. Let $[x y]$ be the common edge of the two faces on $\Delta_{0}^{3} \cap \partial K$.

(2.2.1) If there is not an essential $\partial \Delta^{2}$ in $K$ containing the edge $[x y]$, then $K^{\prime}$ is a triangulation of $M$ and $\xi\left(K^{\prime}\right)=\xi(K)$.

(2.2.2) If there is an essential $\partial \Delta^{2}$ in $K$ containing the edge [xy], then $K^{\prime}$ is a triangulation of another manifold such that $\partial K^{\prime} \neq \emptyset$ and $\xi\left(K^{\prime}\right)=\xi(K)-6$.

By Claim 5.1 and this lemma, we obtain an element $K^{\prime}$ of $\Gamma$ such that $K^{\prime}<K$. This contradicts the minimality of $K$. Thus $K$ does not have a tetrahedron which contains two or more faces on $\partial K$.

Proof of Lemma 5.6. (1) Put $K^{\prime}=\operatorname{cl}\left(K \backslash \Delta_{0}^{3}\right)$ (see Figure 3).

Then $K^{\prime}$ is a triangulation of $M$ and $\xi\left(K^{\prime}\right)=\xi(K)$. Since $b_{1}\left(K^{\prime}\right)=b_{1}(K),\left|\partial K^{\prime}\right|$ $=|\partial K|$, and $T_{0}\left(K^{\prime}\right)=T_{0}(K)-1$, we have $K^{\prime}<K$.

(2.1) Put $K^{\prime}=\operatorname{cl}\left(K \backslash \Delta_{0}^{3}\right)$ (see Figure 4).

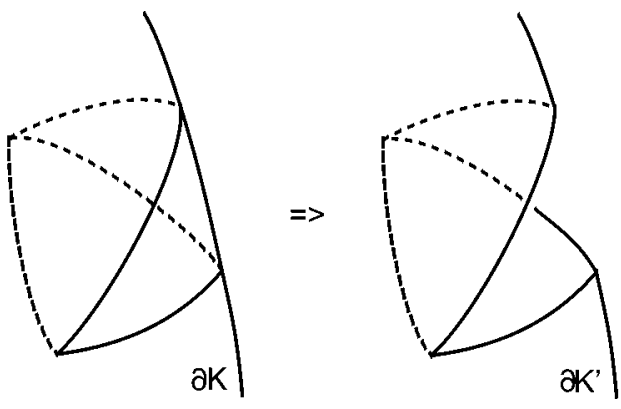

Figure 4 


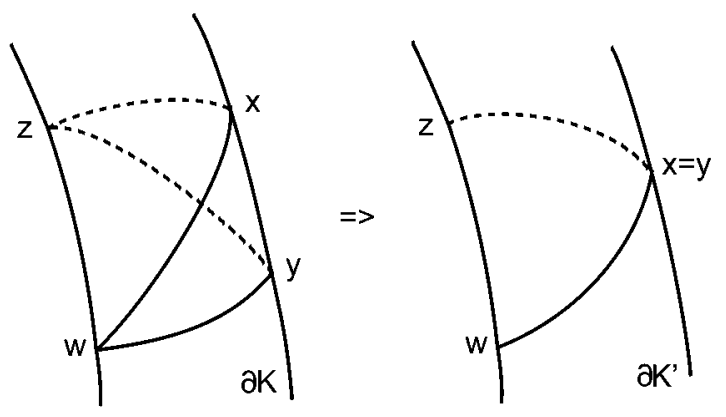

FiguRE 5

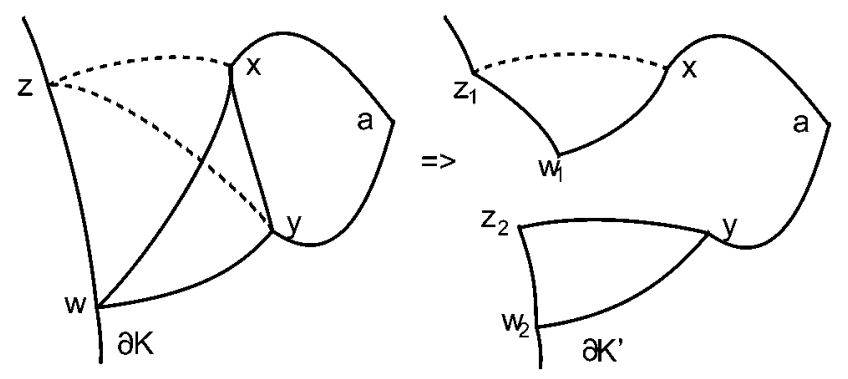

FiguRE 6

Then $K^{\prime}$ is a triangulation of $M$ and $\xi\left(K^{\prime}\right)=\xi(K)-2$. Since $b_{1}\left(K^{\prime}\right)=$ $b_{1}(K),\left|\partial K^{\prime}\right|=|\partial K|$, and $T_{0}\left(K^{\prime}\right)=T_{0}(K)-1$, we have $K^{\prime}<K$.

(2.2.1) Let $K^{\prime}$ be the cell complex obtained from $K$ by contraction of the edge $[x y]$ (see Figure 5).

Since $\partial K \neq \emptyset, K$ does not contain an essential $\partial \Delta^{4}$. By the assumption, $K$ contains neither an essential $\partial \Delta^{3}$ nor an essential $\partial \Delta^{2}$ containing $[x y]$. Thus $K^{\prime}$ is a triangulation by Lemma 4.2 , and we have $\xi\left(K^{\prime}\right)=\xi(K)$. Since $b_{1}\left(K^{\prime}\right)=$ $b_{1}(K),\left|\partial K^{\prime}\right|=|\partial K|$, and $T_{0}\left(K^{\prime}\right)=T_{0}(K)-1$, we have $K^{\prime}<K$.

(2.2.2) To obtain another triangulation $K^{\prime}$, we take the closure of $K \backslash \Delta_{0}^{3}$ and separate two faces $[x z w]$ and $[y z w]$ by dividing the edge $[z w]$ into two copies (see Figure 6).

Then $K^{\prime}$ is a triangulation which satisfies $\partial K^{\prime} \neq \emptyset$ and $\xi\left(K^{\prime}\right)=\xi(K)-6$. Since $b_{1}\left(K^{\prime}\right)=b_{1}(K)-1$, we have $K^{\prime}<K$.

5.4. Proof of Claim 5.4. Let $\mu_{i}(K)$ be $\left(\sum_{e \in \text { int } K}\right.$ order $\left.e\right) / E_{i}(K)$ or 0 according to whether $E_{i}(K) \neq 0$ or not. Put

$$
\mu_{\partial}^{i}(K)=\left(\sum_{e \in \partial K} \sharp\{\text { face } f \in \operatorname{int} K \mid f \succ e\}\right) / E_{\partial}(K) .
$$

Then either $E_{i}(K)=0$ or $\mu_{i}(K) \geq 4$ by Claim 5.2. By Claim 5.3, any edge of $\partial K$ is contained in at least one face of $i n t K$. Thus we have $\mu_{\partial}^{i}(K) \geq 1$. Since $\partial K$ is a triangulation of a closed 2-manifold, $3 F_{\partial}(K)=2 E_{\partial}(K)$. By the definitions of 
$\mu_{i}(K)$ and $\mu_{\partial}^{i}(K), 3 F_{i}(K)=\mu_{i}(K) E_{i}(K)+\mu_{\partial}^{i}(K) E_{\partial}(K)$. Hence we have

$$
\begin{aligned}
\xi(K) & =3 F_{0}(K)-4 E_{0}(K) \\
& =3\left(F_{i}(K)+F_{\partial}(K) / 2\right)-4\left(E_{i}(K)+E_{\partial}(K) / 2\right) \\
& =3 F_{i}(K)-4 E_{i}(K)-E_{\partial}(K) \\
& =\left(\mu_{i}(K)-4\right) E_{i}(K)+\left(\mu_{\partial}^{i}(K)-1\right) E_{\partial}(K) \geq 0 .
\end{aligned}
$$

\section{Proof of the EQUality CASes}

First we prove the latter half of (a). Let $K$ be a triangulation of $B^{3}$ with $\xi(K)=$ -6 . Suppose $K$ has $\Sigma$, an essential $\partial \Delta^{3}$. If $\Sigma=\partial K$, put $K^{\prime}=K \cup_{\Sigma} \Delta^{3}$ as in Lemma 4.3 (1). By Lemma $4.3(1)$ and Lemma $4.5, \xi(K)=\xi\left(K^{\prime}\right)+6>-6$, a contradiction. Thus $\Sigma \neq \partial K$. Since $K$ is a triangulation of $B^{3}, \Sigma$ is a triangulation of a separating 2-sphere. Let $K_{1}$ and $K_{2}$ be as in Lemma 4.3 (2). Then $K_{1}$ is a triangulation of $S^{3}$ and $K_{2}$ is a triangulation of $B^{3}$. By Lemma $4.3(2)$, Lemma 4.5, and the inequality part of Theorem 3.1, $\xi(K)=\xi\left(K_{1}\right)+\xi\left(K_{2}\right)+12>-12+(-6)+12=-6$, a contradiction. Therefore $K$ does not have an essential $\partial \Delta^{3}$.

Suppose $K$ contains an edge of order 3 . Since $K$ does not have an essential $\partial \Delta^{3}$, there is a triangulation $K^{\prime}$ such that $\xi\left(K^{\prime}\right)=\xi(K)-2=-8$ by Lemma 5.5. This contradicts the inequality part of Theorem 3.1 (a). Thus $K$ satisfies the conclusion of Claim 5.2.

If $K$ satisfies the conclusion of Claim 5.3, then $\xi(K) \geq 0$ by Claim 5.4, a contradiction. Thus $K$ has a tetrahedron which has two or more faces on $\partial K$, and hence either $K=\Delta^{3}$, or we can reduce $K$ by the operations in the proof of Lemma 5.6. Since the only such operations that could give equality are those illustrated in Figures 3 and 5, we have $K=\natural^{k} \Delta^{3}$ for some $k$. This completes the proof of Theorem 3.1 (a).

Next we prove the equality part of Theorem 3.1 (b). Let $M \neq B^{3}$, and let $K$ be a triangulation of $M$ with $\xi(K)=0$. Suppose $K$ has $\Sigma$, an essential $\partial \Delta^{3}$. If $\Sigma \subset \partial K$, put $K^{\prime}=K \cup_{\Sigma} \Delta^{3}$ as in Lemma $4.3(1)$. By Lemma $4.3(1), \xi\left(K^{\prime}\right)=\xi(K)-6=-6$. If $\partial K^{\prime}=\emptyset$, then $K^{\prime}$ is a triangulation of $S^{3}$ by Lemma 4.5 and hence $K$ is a triangulation of $B^{3}$, a contradiction. Thus $\partial K^{\prime} \neq \emptyset$, and hence we have $K^{\prime}=\natural^{k} \Delta^{3}$ by Theorem 3.1 (a). This contradicts the fact that int $K^{\prime}$ has the image of $\Sigma$. Therefore $\Sigma$ is not contained in $\partial K$.

Suppose $\Sigma$ is a triangulation of a non-separating 2-sphere. Let $K^{\prime}$ be as in Lemma $4.3(2)$. Then $\xi\left(K^{\prime}\right)=\xi(K)-12=-12$. This contradicts the inequality part of Theorem 3.1 (a). Thus $\Sigma$ is a triangulation of a separating 2-sphere. Put $K^{\prime}=K_{1} \sqcup K_{2}$ as in Lemma $4.3(2)$. Since $\xi\left(K_{1}\right)+\xi\left(K_{2}\right)+12=\xi(K)=0$, we have $\xi\left(K_{i}\right)=-6$ for $i=1,2$, and hence $K_{i}=\natural^{k} \Delta^{3}$. But we cannot take all the faces of $\Sigma_{1}$ in $i n t K_{1}$, a contradiction. Therefore $K$ does not contain an essential $\partial \Delta^{3}$.

Case 1. Suppose $K$ satisfies the conclusion of Claims 5.2 and 5.3. Then $\mu_{i}(K) \geq 4$ and $\mu_{\partial}^{i}(K) \geq 1$. Since $0=\xi(K)=\left(\mu_{i}(K)-4\right) E_{i}(K)+\left(\mu_{\partial}^{i}(K)-1\right) E_{\partial}(K)$ and since $E_{\partial}(K) \neq 0$, we have $\mu_{\partial}^{i}(K)=1$ and either $\mu_{i}(K)=4$ or $E_{i}(K)=0$.

Subcase 1.1. Suppose $E_{i}(K) \geq 1$. Then $\mu_{i}(K)=4$, and we have the following.

(6.1) For each edge $e$ in $i n t K, l k(e)$ consists of 4 edges.

(6.2) For each edge $e$ on $\partial K, l k(e)$ consists of 2 edges.

Here $l k(e)$ is the link complex of an edge $e$. 


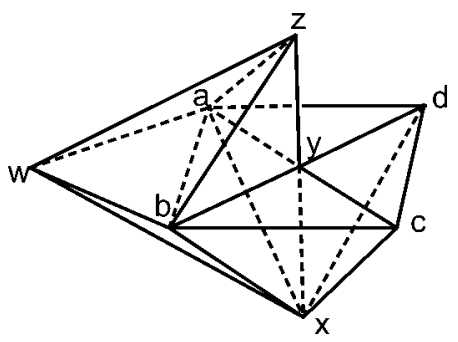

(a)

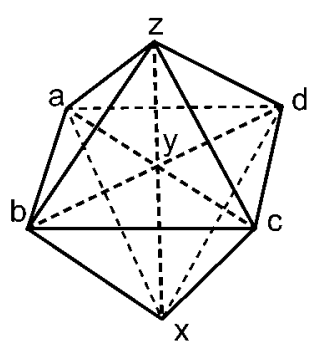

(b)

FIGURE 7

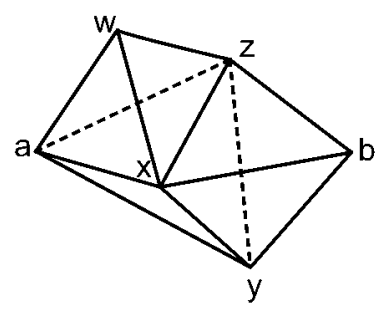

FiguRE 8

Let $[x y]$ be an edge in $i n t K$ and $\{a, b, c, d\}$ the 4 vertices of $l k([x y])$ in this cyclic order. Suppose some edge of $l k([x y])$, say $[a b]$, is in $i n t K$. By $(6.1), l k([a b])$ has 4 vertices; let $\{x, y, z, w\}$ be the 4 vertices in this cyclic order (see Figure 7 (a)). Since $l k([b x])$ contains $[w a],[a y]$, and $[y c],[b x]$ does not lie in $\partial K$ by (6.2). Thus $l k([b x])$ contains $[c w]$ by $(6.1)$, that is, $K$ contains a tetrahedron $[b c w x]$. Since $l k([b y])$ contains $[z a],[a x]$, and $[x c],[b y]$ does not lie in $\partial K$ by $(6.2)$. Thus $l k([b y])$ contains $[c z]$ by $(6.1)$, that is, $K$ contains a tetrahedron $[b c y z]$. Therefore $l k([b c])$ contains $[w x],[x y]$, and $[y z]$, and hence $[b c]$ is in $i n t K$ by (6.2). Similarly, we see that $[c d]$ and $[d a]$ are in intK. Thus $K$ contains the join of $l k([x y])$ and $l k([a b])$, and hence $\partial K=\emptyset$, a contradiction. Therefore any edge of $l k([x y])$ is on $\partial K$.

By $(6.2), l k([a b])$ consists of two edges $[x y]$ and, say, $[y z]$. For reasons similar to the above, $[a y]$ and $[b y]$ lie in $i n t K$. This implies that $[c y]$ and $[d y]$ also lie in intK. Thus $K$ contains the join of $\{[x y],[y z]\}$ and $l k([x y])$ (see Figure 7 (b)). If $K$ contains another tetrahedron, say $[a b z w]$, then $l k([a b])$ contains $[x y],[y z]$, and $[z w]$, and hence $[a b]$ lies in int $K$, a contradiction. Thus $K$ is the join of $\{[x y],[y z]\}$ and $l k([x y])$. This contradicts the hypothesis that $K$ is not a triangulation of $B^{3}$.

Subcase 1.2. Suppose $E_{i}(K)=0$. Since $K \neq \Delta^{3}, K$ has at least two tetrahedra, say $[a x y z]$ and $[x y z b]$, with a common face. Since $\mu_{\partial}^{i}(K)=1, K$ satisfies the condition (6.2). Since $l k([a x])$ contains 2 edges $[y z]$ and, say, $[z w]$, by $(6.2), K$ contains a tetrahedron $[a x z w]$ (see Figure 8$)$. Thus $l k([x z])$ contains $[w a],[a y]$, and $[y b]$, and hence $[x z]$ is not on $\partial K$ by $(6.2)$, a contradiction.

Case 2. Suppose $K$ does not satisfy the conclusions both of Claim 5.2 and of Claim 5.3. Since $K \neq \Delta^{3}, K$ can be reduced by the operations in the proof of Lemma 5.5 or Lemma 5.6. 
Suppose $K$ can be reduced by the operation in Figure 2 or 4 . Then we can obtain a triangulation $K^{\prime}$ of $M \neq B^{3}$ satisfying $\xi\left(K^{\prime}\right)=\xi(K)-2=-2$. This contradicts the inequality part of Theorem 3.1 (b).

Suppose $K$ can be reduced by the operation in Figure 3 or 5 . Since each operation does not change the topology of the manifold and $\xi\left(K^{\prime}\right)=\xi(K)=0, K^{\prime}$ does not satisfy the conclusions both of Claim 5.2 and of Claim 5.3. This implies that we can construct a finite sequence of such operations from $K$ to $K_{0}$ which is a triangulation of $M \neq B^{3}$ satisfying $\xi\left(K_{0}\right)=0$ and which can be reduced neither as in Figure 3 nor as in Figure 5.

Since the operation in Figure 6 decreases $\xi$ by $6, K_{0}$ can be reduced to $K_{0}^{\prime}$ which satisfies $\xi\left(K_{0}^{\prime}\right)=\xi\left(K_{0}\right)-6=-6$. By Theorem 3.1 (a), $K_{0}^{\prime}=\natural^{k} \Delta^{3}$. Since $K_{0}$ can be obtained from $K_{0}^{\prime}$ by a 1-handle addition and $K$ can be obtained from $K_{0}$ by the inverse operations in Figure 3 and 5 , it follows that $K=\natural^{k} \Delta^{3} / \sim$ and $M=D^{2} \times S^{1}$ or $D^{2} \tilde{\times} S^{1}$. This completes the proof of Theorem 3.1.

\section{ACNOWLEDGMENTS}

The author would like to sincerely thank Professors K. Kawakubo, M. Sakuma and T. Kobayashi for their helpful suggestions.

\section{REFERENCES}

1. F. Luo and R. Stong, Combinatorics of triangulations of 3-manifolds, Trans. Amer. Math. Soc. 337 (1993), 891-906. MR 93h:57035

2. C. P. Rourke and B. J. Sanderson: Introduction to Piecewise-Linear Topology, Springer, Berlin, 1972. MR 50:3236

3. M. Tamura, The average edge order of triangulations of 3-manifolds, Osaka J. Math. 33 (1996), 761-773. CMP 97:05

4. D. Walkup, The lower bound conjecture for 3- and 4-manifolds, Acta Math. 125 (1970), 75-107. MR 43:1038

Department of Mathematics, Osaka University, Toyonaka, Osaka 560, Japan

E-mail address: tamura@klingon.math.wani.osaka-u.ac.jp 